\title{
Les modèles physiques en hydraulique urbaine
}

\author{
Physical models in urban hydraulics \\ par M. Martaud, S. Heywood \\ SAFEGE Ingénieurs Conseils, Hydraulic Models Limited
}

Physical models are a means of in-depth investigation. In Urban Hydraulics they can be used for most types of infrastructure, including pumping stations, treatment facilities and the more complex sewer system components. Models can serve not only to check and adjust the design of new works prior to their construction but also to determine the exact causes of impaired operation of existing facilities and propose solutions for their rehabilitation - solutions which will have been tried and tested beforehand on the model.

This paper outlines the mathematical equations underlying model design, demonstrating, in the process, the constraints set by Physical Science on the design, construction and use of the model, which will be crucial to the success of the investigation. The procedure generally adopted in a model-based study is then described, together with recommendations on suitable model materials and on the contents of the report to be submitted to the client.

The final part of this paper deals with pumping stations, treatment works and the more complex network components, respectively. The problems specific to each type of facility are described as well as particular aspects of the modelling procedure on their corresponding models.

\section{INTRODUCTION}

Le maître d'ouvrage qui commandite l'étude sur modèle physique d'un ouvrage d'hydraulique urbaine est assuré de la réussite technique de son opération : le projet proposé par son maître d'œuvre sera validé en l'état ou amendé jusqu'à ce que les performances souhaitées soient atteintes. Au stade ultérieur de la réalisation, le titulaire du marché aura l'assurance de réaliser une installation dont le fonctionnement hydraulique ne souffrira pas de mises au point, parfois difficiles à faire, voire d'efficacité incertaine, au moment de la réception de l'ouvrage.

En effet, on observe souvent des désordres hydrauliques sur des ouvrages conçus uniquement en appliquant les règles élémentaires énoncées dans les manuels. Ce constat a conduit SAFEGE à proposer, en partenariat avec Hydraulic Models Limited, des études détaillées d'ouvrages neufs ou existants d'hydraulique urbaine.

Le modèle physique appliqué à l'hydraulique urbaine peut être utilisé dans les projets de tous les types d'ouvrage (stations de pompage, bassins de traitement d'eau, déversoirs d'orage, siphons, etc...). Son coût doit être comparé, suivant les cas, aux économies de coût d'exploitation (amélioration des rendements, réduction des causes de désordres causant des usures prématurées d'équipements), aux économies de coût d'investissement (optimisation des plans) ou aux coûts des interminables ajustements en phase de réception.

Le modèle physique intervient, dans le déroulement d'une étude, dès que le dessin de l'ouvrage est bien cerné et, au plus tard, au stade des études détaillées précédant la rédaction du CCTP.

Le recours au modèle physique en phase de conception apporte ainsi très tôt dans l'opération la garantie de résultats qui affermit la valeur technique d'un projet. Cette assurance est nécessaire dans tous les contextes :

- pour des ouvrages neufs, complexes et (ou) coûteux [1] ; - pour des ouvrages dont le bon fonctionnement est stratégique (soit au plan du service rendu au public soit au plan économique) ;

- pour des ouvrages existants dont on prévoit des extensions ou des modifications ;

- pour des ouvrages existants qui présentent des problèmes d'exploitation récurrents que l'expérience de terrain ne parvient pas à résoudre.

Cet article illustre l'application de ces principes à des ouvrages réalisés récemment en France et à l'étranger. 


\section{II —CATÉGORIE DE MODÈLES EM- PLOYÉS EN HYDRAULIQUE URBAINE}

En hydraulique urbaine, les modèles physiques sont généralement des modèles similaires à fond fixe. Le modèle est dit similaire lorsque les échelles géométriques de réduction horizontale et verticale sont égales entre elles. En revanche, lorsqu'il existe au moins une échelle géométrique différente des autres, le modèle est dit distordu.

Les limites matérielles d'un modèle physique et, particulièrement le fond du modèle, peuvent être fixes ou mobiles. Les modèles à fonds mobiles sont ceux qui s'appliquent à l'étude de la stabilisation des lits de rivière, des plages, etc... Ils combinent la représentation de l'écoulement, de la sédimentation et du transport solide. Les modèles à fonds fixes sont destinés à l'étude des phénomènes où la reproduction fidèle des écoulements est primordiale. Ce sont ces derniers qui sont employés en hydraulique urbaine.

Tous les modèles peuvent être employés pour simuler le comportement du prototype $\left.{ }^{1}{ }^{1}\right)$ indépendamment du temps (régime stationnaire) ou en fonction du temps (régime instationnaire).

\section{III —CONTRAINTES DE RÉALISATION DU PROTOTYPE À L'ÉCHELLE}

\subsection{Similitudes géométrique, cinématique et dynamique}

Le modèle physique doit être semblable au prototype. Cette exigence impose que soient respectées les similitudes géométrique, cinématique et dynamique entre le modèle et le prototype. La similitude géométrique est la relation qui existe entre les longueurs, pour un modèle similaire elle est plus banalement appelée échelle.

La similitude cinématique est obtenue lorsque le champ de vitesse du modèle représente fidèlement celui du prototype. C'est-à-dire que le rapport des vitesses observées en deux points homologues du prototype et du modèle doit être constant.

$$
\frac{V_{p}}{V_{m}}=E_{v}
$$

Notations :

- $V_{p}:$ vitesse dans le prototype ;

$-V_{m}^{p}:$ vitesse dans le modèle ;

$-E_{v}^{m}$ : échelle des vitesses.

Plus généralement :

$$
E_{v}=E_{l} \cdot E_{t}^{-1}
$$

Notations :

- $E_{v}$ : échelle des vitesses ;

- $E_{1}$ : échelle des longueurs;

- $E_{t}$ : échelle des temps.

La similitude complète du mouvement du fluide entre le modèle et le prototype ne sera obtenue que lorsque le polygone des forces qui agissent sur une particule de fluide du prototype sera reproduit fidèlement sur le modèle. A titre d'exemple, dans le cas des forces d'inertie, cette exigence se

1 En modélisation physique, le terme prototype est le vocable consacré pour désigner le projet étudié et le modèle est donc le modèle réduit construit en laboratoire. traduit en équation en appliquant l'expression de la loi fondamentale de la dynamique.

$$
\frac{F_{p}}{F_{m}}=E_{m} \cdot E_{l} \cdot E_{t}^{-2}
$$

Notations :

$-F_{p}:$ force dans le prototype ;

$-F_{m}^{p}:$ force dans le modèle ;

- $E_{m}:$ échelle des masses;

- $E_{1}$ : échelle des longueurs ;

$-E_{t}$ : échelle des temps.

\subsection{Contraintes de qualité d'exécution d'un modèle physique}

La lecture des équations du paragraphe précédent montre que ni la similitude cinématique ni la similitude dynamique ne peuvent être obtenues si la similitude géométrique n'est pas respectée. Cette contrainte se traduit très concrètement par l'obligation d'effectuer un travail de conception puis de construction du modèle particulièrement soigné. Ce soin est le garant de la qualité des observations, de la fiabilité des mesures et des conclusions tirées de l'étude sur modèle.

Respecter la similitude des masses exige également du laboratoire une dextérité confirmée dans la conception et la réalisation des accessoires du modèle. C'est une contrainte qui s'ajoute à la précédente dans les études où interviennent par exemple des objets sur lesquels s'appliquent des efforts d'origine hydrodynamique : étude de rivières (avec des embarcations) pour les parcs d'attraction ou étude d'engins de curage de canaux ou d'égout.

Enfin, respecter l'échelle des temps est une contrainte qui s'applique à l'instrumentation et à l'équipement de contrôle du fonctionnement du modèle mais aussi à l'expérimentateur. Cependant, il faut noter que cette échelle n'intervient pas dans les études en régime stationnaire.

Le modèle physique se révèle donc être bien plus qu'une maquette. C'est, en réduction dans le temps et dans l'espace, une sorte de répétition de la construction puis de l'exploitation de l'ouvrage projeté. Le modèle se conçoit avant que d'être construit, on en dessine donc les plans de conception puis d'exécution. Ceux-ci sont validés avant l'exécution proprement dite. Le modèle est ensuite construit par des spécialistes puis exploité par des expérimentateurs confirmés.

\section{G CRITÈRES DE SIMILITUDE PHY- SIQUE POUR CONCEVOIR UN MODÈLE}

\subsection{Rapports de similitude des forces intervenant dans les écoulements}

Respecter la similitude dynamique place l'ingénieur devant la situation suivante : le prototype et le modèle n'ont pas les mêmes dimensions géométriques mais ils doivent être le siège des mêmes phénomènes physiques. L'ingénieur doit donc connaître ces phénomènes physiques pour les réduire convenablement à l'échelle avant de commencer la conception de son modèle. Il faut donc pouvoir dresser un inventaire correct des forces qui agissent sur le fluide et déclenchent les phénomènes étudiés.

La capacité de diagnostic, tirée de l'expérience, est donc une des clés de la réussite d'une étude sur modèle physique. Elle permet de dominer le projet au point de pouvoir dresser par avance la liste des phénomènes physiques qui pourraient limiter l'efficacité de l'ouvrage ou s'opposer à son bon fonc- 
tionnement. Selon la nature de l'ouvrage étudié, cette capacité de diagnostic oriente l'attention vers un groupe de phénomènes physiques et permet ainsi de connaître, par les équations qui les régissent, les paramètres qui entrent en jeu dans ces phénomènes. En d'autres termes, l'expérience permet de sélectionner les forces dont l'action est prépondérante dans le fonctionnement hydraulique de l'ouvrage.

En hydraulique, les principales forces qui interviennent sont :

- les forces d'inertie ;

- les forces de turbulence;

- les forces de viscosité ;

- l'action de la pesanteur ;

- la capillarité.

On démontre rapidement [2] que le rapport de similitude change pour chacune de ces catégories :

- pour les forces d'inertie (cf. paragraphe 3.1 );

$$
\frac{F_{p}}{F_{m}}=E_{m} \cdot E_{l} \cdot E_{t}^{-2}
$$

- pour les forces de turbulence ;

$$
\frac{F_{p}}{F_{m}}=E_{m} \cdot E_{l} \cdot E_{t}^{-2}
$$

- pour les forces de viscosité ;

$$
\frac{F_{p}}{F_{m}}=E_{\mu} \cdot E_{l}^{2} \cdot E_{t}^{-1}
$$

- pour l'action de la pesanteur;

$$
\frac{F_{p}}{F_{m}}=E_{\rho} \cdot E_{l}^{3} \cdot E_{g}
$$

Notations :

$-F_{p}:$ force dans le prototype ;

$-F_{m}^{p}:$ force dans le modèle ;

- $E_{m}$ : échelle des masses;

$-E_{1}^{m}$ : échelle des longueurs :

- $E_{m}$ : échelle des viscosités dynamiques;

- $E_{r}^{m}$ : échelle des masses volumiques ;

- $E_{g}$ : échelle des accélérations de la pesanteur ;

$-E_{t}^{g}$ : échelle des temps.

\subsection{Nombres adimensionnels}

Suivant la nature de l'écoulement considéré, les diverses catégories de forces citées précédemment interviennent d'une manière plus ou moins importante. A ce stade, il convient de faire usage des nombres adimensionnels qui expriment l'importance relative de deux phénomènes comparés l'un à l'autre. Les forces d'inertie ne peuvent jamais être négligées en hydraulique puisqu'il y a toujours une masse liquide en mouvement. Le Tableau 1 dresse une liste des principaux nombres où interviennent ces forces.
Tableau 1. - Liste des principaux nombres adimensionnels.

\begin{tabular}{|ll|}
\hline Nombre & Caractéristiques \\
\hline Euler & $\begin{array}{l}\text { Relation entre les forces d'inertie } \\
\text { et la pression }\end{array}$ \\
Froude & $\begin{array}{l}\text { Relation entre les forces d'inertie } \\
\text { et l'action de la pesanteur }\end{array}$ \\
Reynolds & $\begin{array}{l}\text { Relation entre les forces d'inertie } \\
\text { et la viscosité }\end{array}$ \\
Weber & $\begin{array}{l}\text { Relation entre les forces d'inertie } \\
\text { et la tension superficielle }\end{array}$ \\
Cauchy & $\begin{array}{l}\text { Relation entre les forces d'inertie } \\
\text { et l'élasticité }\end{array}$ \\
Rossby & $\begin{array}{l}\text { Relation entre les forces d'inertie } \\
\text { et la force de Coriolis }\end{array}$ \\
Richardson & $\begin{array}{l}\text { Relation entre les forces d'inertie } \\
\text { et les forces d'origine thermodynamique }\end{array}$ \\
Strouhal & $\begin{array}{l}\text { Relation entre les forces d'inertie } \\
\text { et les forces générées par des phéno- }\end{array}$ \\
& mènes périodiques dans l'écoulement
\end{tabular}

L'équation (4) ci-dessus montre que le rapport de similitude des forces d'inertie est le même que celui des forces de turbulence, ce qui explique l'absence de nombre adimensionnel particulier pour ce cas. Les forces d'inertie et de turbulence sont intrinsèquement de même nature.

On montre [2] [3] que c'est l'opération qui consiste à sélectionner les forces dont l'action est prépondérante qui conduit à identifier le nombre adimensionnel dont les valeurs sont les mêmes dans le modèle et dans le prototype. C'est l'habitude de l'expérimentateur averti qui a consacré le raccourci où l'on annonce tout de suite le nombre adimensionnel employé pour la similitude physique.

\subsection{Incompatibilité des similitudes physiques}

Malheureusement, on montre aussi que le choix d'une similitude physique (identifiée par le nom du nombre adimensionnel opportunément retenu pour le projet de modélisation) conduit à exclure de fait les autres similitudes. Le Tableau 2, établi à partir de M. A VERGARA SANCHEZ [3] en faisant l'hypothèse que les fluides utilisés dans le modèle et dans le prototype sont les mêmes, montre que les principales similitudes physiques sont incompatibles entre elles.

\section{$V \square$ APPLICATION AUX MODÈLES RÉDUITS D'HYDRAULIQUE URBAINE}

\subsection{Similitudes physiques usuelles}

Les deux similitudes les plus employées sont celle de Froude et celle de Reynolds. La première est employée pour

Tableau 2. - Comparaison de quatre conditions de similitude [3].

\begin{tabular}{|lllll|}
\hline Echelles & Similitude de Froude & Similitude de Reynolds & Similitude de Weber & Similitude de Cauchy \\
\hline Longueur & $E_{1}$ & $E_{1}$ & $E_{1}$ & $E_{1}$ \\
Temps & $E_{1}^{1 / 2}$ & $E_{1}^{2}$ & $E_{1}^{3 / 2}$ & $E_{1}$ \\
Vitesse & $E_{1}^{1 / 2}$ & $E_{1}^{-1}$ & $E_{1}^{-1 / 2}$ & 1 \\
Débits & $E_{1}^{5 / 2}$ & $E_{1}$ & $E_{1}^{3 / 2}$ & $E_{1}^{2}$ \\
Forces & $E^{3}$ & 1 & $E_{1}^{-1}$ & $E_{1}^{2}$ \\
Pressions & $E_{1}$ & $E_{1}^{-2}$ & $E_{1}^{-1}$ & 1 \\
\hline
\end{tabular}


des écoulements à surface libre généralement très turbulents où les forces de viscosité sont négligeables devant l'action de la pesanteur et de la turbulence. La similitude de Reynolds est employée pour les écoulements en charge tant que la tension superficielle joue un rôle négligeable ou tant que la turbulence et l'inertie ne prennent pas une place prépondérante dans l'écoulement. Cette similitude est aussi employée pour étudier la résistance opposée par un corps entièrement noyé.

\subsection{Seuil de Reynolds}

On peut étendre la similitude de Froude à des écoulements en charge tant que les expériences se situent au-delà du seuil de Reynolds dont la définition est donnée dans les paragraphes qui suivent.

La perte de charge dans un ouvrage quelconque fonctionnant en charge s'exprime par la relation :

$$
J=\frac{\lambda^{*}}{D} \cdot \frac{U^{2}}{2 \cdot g}
$$

Notations :

- $J$ : perte de charge $(\mathrm{m} / \mathrm{m})$

$-\lambda^{*}$ : coefficient de perte de charge ;

- $D$ : diamètre hydraulique de la section de passage $(\mathrm{m})$;

- $U$ : vitesse d'écoulement dans la section $(\mathrm{m} / \mathrm{s})$;

$-g$ : accélération de la pesanteur $\left(9,81 \mathrm{~m} / \mathrm{s}^{2}\right)$.

Le coefficient $\lambda *$ est adimensionnel et dépend du nombre de Reynolds (noté $R$ dans la suite de ce texte) et de la rugosité relative des parois. La similitude des écoulements dans le prototype et sur le modèle impose que $\lambda^{*}$ soit le même sur le prototype et sur le modèle.

Le diagramme $\lambda^{*}=f(R)$ montre que pour une rugosité relative donnée, $\lambda^{*}$ est constant au-delà d'une valeur donnée de $R\left({ }^{2}\right)$. Cette valeur du nombre de Reynolds est appelée seuil de Reynolds.

Le coefficient $\lambda^{*}$ sera donc identique sur le modèle et sur le prototype si les écoulements sont tels que le seuil de Reynolds soit dépassé sur le prototype et sur le modèle. L'essai sur le modèle pourra alors être réalisé en employant la similitude de Froude même si les nombres de Reynolds ne sont pas les mêmes dans le modèle et dans le prototype.

Toutefois, si le nombre de Reynolds approche, sur la maquette, la frontière du domaine de transition entre les régimes laminaire et turbulent, un écoulement laminaire pourrait s'établir sur la maquette alors que sur le prototype on pourrait observer un régime transitoire ou turbulent. Cela est inacceptable et il faut alors choisir un nombre de Reynolds minimum pour conduire les expériences.

La valeur du seuil de Reynolds varie d'un auteur à un autre. M. Carlier [2] cite une valeur minimale de 4000 alors que M. S. Yalin [4] propose $R=10000$. C'est la valeur du seuil de Reynolds qui détermine la limite inférieure de l'échelle de réduction géométrique d'un modèle physique.

\subsection{Prise en compte de la rugosité des parois}

La vitesse d'écoulement dans un canal siège d'un écoulement à surface libre, en régime uniforme, s'exprime par :

$$
U=K \cdot R^{(2 / 3)} \cdot \sqrt{I}
$$

2 Cette zone du diagramme universel $\lambda^{*}=f(R)$ s'appelle la harpe de Nikuradse.
Notations :

- $U$ : vitesse d'écoulement dans la section $(\mathrm{m} / \mathrm{s})$;

- $K$ : coefficient de Gaukler Manning $\left(\mathrm{m}^{1 / 3} . \mathrm{s}\right)$;

- I: pente du chenal $(\mathrm{m} / \mathrm{m})$;

$-R$ : rayon hydraulique de la section de passage $(\mathrm{m})$.

La vitesse d'écoulement dans le modèle, en régime uniforme, s'exprime par :

$$
U_{m}=K_{m} \cdot R_{m}^{(2 / 3)} \cdot \sqrt{I}
$$

Notations :

$-U_{m}$ : vitesse d'écoulement dans la section du modèle $(\mathrm{m} / \mathrm{s}) ;$

$-K_{m}$ : coefficient de Gaukler-Manning sur le modèle $\left(\mathrm{m}^{1 / 3} \cdot \mathrm{s}\right)$;

- $I:$ pente du chenal $(\mathrm{m} / \mathrm{m})$;

$-R_{m}$ : rayon hydraulique de la section de passage sur le modèle $(\mathrm{m})$;

Le rapport membre à membre de ces deux équations conduit à l'égalité :

$$
K_{m}=K \cdot E_{l}^{(1 / 6)}
$$

Pour respecter la similitude de pertes de charge linéaires et les lignes d'énergie, il faut donc adopter un coefficient de Gaukler-Manning (qui n'est pas un paramètre sans dimension) sur le modèle calculé par application de l'équation précédente. On constate que l'échelle géométrique intervient à la puissance $1 / 6$, les variations de la rugosité du prototype sont estompées par le passage sur le modèle réduit.

Le calage du modèle numérique du réseau d'assainissement de Paris, effectué par SAFEGE dans le cadre du diagnostic de ce réseau, a conduit à fixer à $K$ la valeur $55 \mathrm{~m}^{1 / 3}$.s. Pour un modèle à l'échelle $1 / 5\left(E_{1}=5\right)$ d'une portion de collecteur de ce réseau, le coefficient $K_{m}$ doit donc être égal à $72 \mathrm{~m}^{1 / 3} \mathrm{~s}$.

Dans la pratique, il est difficile de maîtriser la réalisation des modèles au point de reproduire convenablement la rugosité. Il est donc préférable de déterminer cette dernière par l'étalonnage du modèle.

\subsection{Notion d'effet d'échelle}

L'effet d'échelle est un phénomène parasite qui limite l'utilisation de la similitude et donc la validité des mesures et des observations faites sur le modèle réduit. C'est le cas par exemple si le seuil de Reynolds défini au paragraphe 5.2 n'est pas respecté (différence de régime d'écoulement entre le prototype et le modèle).

$\mathrm{Si}$ la profondeur du modèle devient trop petite à la suite du choix d'une échelle géométrique inadaptée, la tension superficielle peut prendre une importance non négligeable (alors que son rôle avait été négligé au stade du choix de la similitude physique employée).

\subsection{Choix d'une échelle pour un modèle}

L'échelle correcte, connue a priori, pour les modèles physiques n'existe donc pas. Le choix de l'échelle dépend donc de nombreuses contraintes qu'il faut concilier. Il existe plusieurs échelles possibles pour un même modèle, de l'échelle minimale imposée notamment par le seuil de Reynolds jusqu'à l'échelle 1 (pas de réduction donc pas d'effets d'échelle). L'échelle employée est souvent un compromis entre l'efficacité des expériences (qualité des observations, accessibilité au modèle, etc...) et les contraintes techniques et économiques du laboratoire : disponibilité d'espace au sol, localisation et caractéristiques des installations connexes, etc... 


\section{ANATOMIE D'UN ESSAI SUR MO- DÈLE PHYSIQUE}

\subsection{Quand lancer l'étude sur modèle physique?}

Il est évident que le modèle physique ne peut pas être employé trop tôt dans le déroulement des études du maître d'cuvre : certaines contraintes, en particulier les contraintes d'accès aux ouvrages pour l'entretien, apparaissent relativement tardivement dans la phase de conception et conduisent parfois à de nets remaniements du dessin. Pourtant, il ne faut pas trop tarder pour effectuer l'étude sur modèle car c'est lorsque le dessin est encore peu figé que l'on peut procéder aux remaniements des infrastructures pour rechercher des solutions plus économiques en optimisant la taille des ouvrages. Il existe donc une période optimale pour lancer l'étude sur modèle physique que l'on pourrait situer au cours des études détaillées, vraisemblablement peu après la fin de l'avant-projet sommaire.

Quelle que soit cette période, il est clair que l'étude sur modèle physique appartient au domaine des prestations qui précèdent l'appel d'offres et non pas au lot des études d'exécution confiées aux entreprises ; à ce stade, le dessin est trop figé pour accepter des retouches de fond. En outre, l'étude sur modèle physique permet l'optimisation de la taille des ouvrages, cet objectif n'est pas nécessairement conciliable avec celui des entreprises qui répondent à un appel d'offre : la garantie de résultats ne nécessite pas toujours l'optimisation des dimensions des ouvrages.

L'étude sur modèle physique valide ou corrige le projet du maître d'œuvre ce qui permet au maître d'ouvrage de savoir qu'il propose en appel d'offres un ouvrage hydrauliquement viable. Dans ce contexte, si l'appel d'offres autorise les variantes, le maître d'ouvrage est en droit de demander le même niveau de garantie de résultats que celui assuré par son maître d'œuvre. Il est donc clair qu'une variante majeure concernant l'ouvrage lui-même devra faire l'objet d'un nouveau modèle physique.

\subsection{Conception du modèle}

\subsubsection{Détermination des frontières du domaine modélisé}

On peut distinguer trois domaines d'observation dans lesquels l'expérimentateur intervient pour l'étude d'un ouvrage d'hydraulique urbaine :

- le champ éloigné : il contient les ouvrages d'amenée ;

- le champ proche : il s'agit d'une zone qui entoure l'ouvrage :

- le champ de proximité immédiate : il s'agit de l'intérieur de l'ouvrage.

Le choix des limites matérielles du domaine qui doit être modélisé est fixé avant d'exécuter le modèle. L'auteur du projet doit donc avoir l'intuition des désordres qui pourraient naître de sa conception pour proposer le domaine modélisé d'étendue optimale. Dans cet exercice, l'ingénieur conseil doit être à la fois compétent pour s'autocritiquer et humble pour savoir reconnaître les points faibles de son projet. Il n'existe donc pas de contours standards pour un modèle physique d'ouvrage d'hydraulique urbaine.

Dans ce domaine, les conditions d'écoulement sur (ou dans) l'ouvrage étudié seront très largement influencées par la trajectoire imposée à la veine liquide jusqu'au point d'étude. Les effets inertiels étant prédominants dans les écoulements examinés, les coudes, les protubérances, les accidents de parcours génèrent souvent des phénomènes qui se propagent ou qui sont véhiculés loin en aval. Le domaine modélisé se définit donc à l'aide du plan de l'ouvrage mais aussi du plan des ouvrages amont.
On peut donc imaginer, dans certains cas particuliers, de devoir recourir à des modèles dits "emboîtés ". Il s'agit alors de faire un modèle à grande échelle d'un vaste domaine puis un modèle à plus petite échelle d'un domaine plus restreint. Les résultats obtenus sur le grand modèle servent de conditions aux limites sur le petit. Il n'existe pas d'optimum technico-économique préétabli ce qui renvoie pour chaque ouvrage à la nécessité d'une analyse technique préalable et à un accord avec le maître d'ouvrage.

Cette nécessité peut entrer en conflit avec les mécanismes des marchés publics qui accordent peu de place à la mise au point du cahier des charges après le lancement de l'appel d'offres. Il est pourtant important de comprendre qu'une étude sur modèle peut être mise en défaut parce qu'un phénomène perturbateur, naissant en dehors du domaine modélisé, n'aura pas été analysé. L'importance de cet écueil peut être nettement amoindrie si le recours à la modélisation physique a été présent dans l'esprit du maître d'ouvrage et de son maître d'œuvre dès le début de l'opération. En effet dans ces conditions, la conception de l'ouvrage progresse en même temps que la réflexion qui conduit à la délimitation des trois périmètres définis plus haut: le champ éloigné, le champ $e$, le champ de proximité immédiate, ce qui facilite la détermination du périmètre optimal du domaine modélisé.

\subsubsection{Dessins de conception et d'exécution}

Le modèle est conçu à l'aide des plans remis par le maître d'ouvrage ou par son maître d'œuvre. Il est nécessaire que les plans de conception du modèle et, en particulier, les chaînes de cotes soient remis au commanditaire de l'étude pour vérification et approbation avant que le laboratoire ne dessine les plans de fabrication du modèle.

La simplification du dessin est un exercice qui permet d'alléger les coûts de fabrication du modèle mais il n'a de sens que si l'expérience du modélisateur permet de justifier que ces simplifications ne nuiront pas à l'objet de l'étude. La simplification ne peut pas être employée inconsidérément avec pour seule fin de diminuer le coût du modèle réduit.

\subsection{Construction du modèle}

Les modèles comportent deux parties :

- le modèle réduit de l'ouvrage objet de l'étude ;

- l'équipement connexe tel que les pompes de recirculation, les vannes de contrôle, les appareils de mesure, etc...

Dans le cas des modèles d'hydraulique urbaine construits par Hydraulic Models Ltd. Ces deux parties sont conçues et installées de toutes pièces pour chaque étude. Le dessin et la conception du modèle sont effectués de manière à ce que l'accès aux organes de contrôle soit aisé. Ceci rend toute opération de mesure et de réglage particulièrement simple et participe à la bonne maîtrise des expériences. Enfin, en cas d'expérimentations conduites par le maître d'ouvrage luimême, cet arrangement des installations facilite leur prise en main par un intervenant extérieur au laboratoire.

Les modèles sont installés sur des estrades en bois de $0,80 \mathrm{~m}$ environ de hauteur. Les fonds et les bétons de seconde phase du prototype sont en PVC sur le modèle et toutes les parois sont en Plexiglas. La situation du modèle en élévation, conjuguée avec l'emploi du Plexiglas, offre d'excellentes conditions d'observation à l'expérimentateur, notamment pour analyser les écoulements dans l'épaisseur de la veine liquide.

Le recours au Plexiglas nécessite un équipement de découpe spécifique et traduit une grande maîtrise technique qui s'accorde avec les contraintes analysées au paragraphe 3.2. Les assemblages se font par collage. Ce type de modèle, très didactique, peut aussi être conçu pour être démonté après les études et réinstallé chez le maître d'ouvrage. Cet 
avantage technique peut être mis à profit pour transformer le modèle en un support de formation à l'attention des exploitants.

\subsection{Essais}

Les essais nécessitent de consacrer de nombreuses heures de travail à l'observation. Il s'agit de regarder pour connaître, de connaître pour comprendre, de comprendre pour interpréter et diagnostiquer. La mesure intervient quand l'opérateur a compris ce qu'il observe. A ce stade seulement, il peut faire des relevés de données pertinents qui viendront conforter son diagnostic et serviront ensuite de référence pour contrôler l'efficacité des aménagements testés.

SAFEGE et Hydraulic Models Ltd. proposent des aménagements sélectionnés au cours de réunions techniques entre hydrauliciens et génie civilistes pour tenir compte de la faisabilité des solutions envisagées. Ces propositions sont soumises au client pour avis et approbation avant d'être testées. Cette seconde phase d'essai se déroule alors comme la précédente : l'observation minutieuse précède la mesure. La comparaison entre la situation initiale et la situation obtenue après les retouches permet de juger de la qualité des aménagements examinés et de les hiérarchiser.

Il est nécessaire que le maitre d'ouvrage prenne part à au moins deux réunions techniques :

- réception de la première phase d'étude : diagnostic du projet dans son état initial ;

- réception du modèle portant les modifications testées et qui contribuent à rendre opérationnel l'ouvrage étudié.

Il est souhaitable cependant que le maître d'ouvrage ou son maître d'œuvre s'implique plus profondément dans l'étude pour s'en approprier les observations et les résultats et pour se familiariser avec l'outil de travail. On peut par exemple suggérer que le maître d'ouvrage ou son représentant assiste à une séance d'assemblage du modèle, à la mise en eau, aux discussions des possibilités d'aménagement ou bien effectue une séance d'expérimentation menée par leurs soins...

\subsection{Synthèse des travaux}

Les essais sur modèle physique constituent une prestation originale où le document visuel occupe une place importante. SAFEGE et Hydraulic Models Ltd. proposent systématiquement un rapport illustré de photographies en couleur. Ces dernières sont des captures d'image effectuées sur la bande vidéo des enregistrements des expériences. Le rapport est illustré dans le texte par des dessins cotés délivrés sous un format informatique lisible par le logiciel AUTOCAD ${ }^{\circ}$. Le client dispose ainsi des plans de conception du modèle et de tous les documents graphiques nécessaires pour procéder aux retouches de son projet.

Le rapport est accompagné d'un diaporama et d'une cassette vidéo commentée. Le diaporama peut être réalisé, à la demande du client, comme un support de conférence, comme un support de formation (à l'exploitation de l'ouvrage) ou comme un résumé de la démarche expérimentale. Il peut être remis entièrement sur support informatique.

Les prises de vues de la cassette vidéo (dont certaines servent à illustrer le rapport : cf. paragraphe précédent) sont enregistrées au laboratoire et le commentaire est dit par un ingénieur. Selon les besoins du maître d'ouvrage, le commentaire est enregistré en studio d'enregistrement, lu par un acteur professionnel dans la langue du maître d'ouvrage et la cassette est préparée au standard audiovisuel du pays du client. Cette cassette, comme le diaporama, peut être réalisée comme un support de conférence ou de formation ou comme un résumé de la démarche expérimentale.

\section{VII — STATIONS DE POMPAGE}

\subsection{Phénomènes perturbateurs à examiner au cours des expériences}

Le concepteur de la roue d'une pompe dessine sa pièce en admettant que le champ de vitesse à l'aspiration de la pompe est uniforme et parfaitement axial. Il en est rarement ainsi dans la réalité, les principales déviations par rapport au champ de vitesse idéal sont de deux types :

- existence d'une composante tangentielle dans le champ de vitesse ;

- fortes variations du module de la vitesse autour de la vitesse moyenne.

Le premier phénomène induit de la prérotation. La performance réelle de la pompe s'écarte alors de la performance théorique annoncée par le courbier. Il est nécessaire de maintenir la valeur de l'angle de prérotation en deçà des limites fixées par le constructeur de la pompe et qui dépendent du type de roue installée sur la machine.

Les variations du module de la vitesse autour de la vitesse moyenne peuvent déséquilibrer l'impulseur des pompes entraînant une perte de performances, des vibrations voire de la cavitation.

Des perturbations du champ de vitesse peuvent aussi donner naissance à des vortex. Un vortex totalement développé peut dégrader considérablement les performances d'une pompe. Il peut créer des vibrations, générer de la cavitation sur l'impulseur. Les vortex avec entraînement d'air peuvent aller jusqu'à désamorcer une pompe.

Certaines bâches sont alimentées en chute. Dans ces conditions, le jet incident perce la surface du plan d'eau et entraîne avec lui une certaine quantité d'air dispersée dans l'écoulement sous forme de bulles. Celles-ci sont entraînées par l'écoulement vers l'aspiration des pompes. Dans la réalité, l'air présent sous forme de bulles peut désamorcer les pompes, les faire vibrer, augmenter leur niveau sonore.

\subsection{Prérotation}

\subsubsection{Mesure}

Sur un modèle hydraulique de station de pompage, la prérotation est mesurée par un moulinet placé dans l'aspiration de la pompe. Ce moulinet est composé de trois ou quatre ailettes dont l'axe coïncide avec celui de l'aspiration de la pompe. La composante tangentielle du vecteur vitesse met le moulinet en rotation. L'angle de prérotation est défini par le système d'équations ci-dessous, il est positif si le mouvement de rotation s'effectue dans le même sens que celui de la roue, il est négatif dans le cas contraire.

$$
\begin{gathered}
V_{t}=\frac{2 \cdot \pi \cdot n}{60} \\
V_{a}=\frac{4 \cdot Q}{\pi \cdot d^{2}} \\
\theta=\operatorname{Arctan}\left(\frac{V_{t}}{V_{a}}\right)
\end{gathered}
$$

Notations :

$-\theta$ : angle de prérotation ;

$-V_{t}$ : vitesse périphérique de rotation de l'ailette du moulinet $(\mathrm{m} / \mathrm{s})$;

- $V_{a}$ : vitesse axiale d'écoulement $(\mathrm{m} / \mathrm{s})$;

- $\mathrm{r} r$ longueur de l'ailette du moulinet $(\mathrm{m})$;

- $n$ : nombre de tours par minute du moulinet

- $Q$ : débit entrant dans l'aspiration de la pompe $\left(\mathrm{m}^{3} / \mathrm{s}\right)$;

$-d$ : diamètre de l'aspiration de la pompe $(\mathrm{m})$. 
La valeur limite de l'angle de prérotation est d'autant plus basse que la vitesse spécifique de la roue de la pompe est élevée. On pourra admettre une valeur de $5^{\circ}$ pour des roues de type centrifuge (vitesses spécifiques inférieures à 70 ou 80 ) alors qu'il faudra se cantonner au-dessous de 1 ou 2 degrés pour des roues à écoulement axial (vitesse spécifique supérieure à 170).

\subsection{Vorticité}

Lorsqu'un vortex apparaît, le théorème de Helmoltz nous rappelle que le phénomène, même s'il est d'apparence modeste, dispose nécessairement de deux points d'ancrage. Dans le cas des stations de pompage le second point est toujours sur la roue de la machine.

Les vortex qui apparaissent à la surface du plan d'eau peuvent se creuser jusqu'à entraîner de l'air dans la pompe. Cette dernière peut alors se désamorcer, vibrer, être bruyante. L'expérience montre aussi que certains vortex ne sont pas visibles depuis la surface, ce sont les vortex immergés qui naissent sur une paroi ou sur un radier ou encore qui vont d'une pompe à une autre pompe. Les vortex immergés peuvent, de leur côté, devenir des vortex cavitants. Dans ce cas, la roue peut être rongée par la poche de cavitation du cœur du vortex.

Les causes d'apparition du vortex sont nombreuses, par exemple lorsque l'écoulement franchit un divergent de raccordement trop largement ouvert (angle au sommet supérieur à $14^{\circ}$ ), on observe le décollement de la veine liquide. Des vortex naissent dans les plans de cisaillement de la veine liquide et sont transportés vers l'aval par le fluide en mouvement. A l'approche des machines, ces vortex pourront devenir dangereux pour les pompes.

La prudence dicte clairement à l'expérimentateur de faire disparaître les causes qui génèrent les vortex et non de se limiter à en diminuer l'intensité. Le modèle physique est un outil précieux pour cette opération. Toutefois, certains vortex sont susceptibles d'être affectés par les effets d'échelle. Il faut retenir que dans la réalité la transition entre une dépression de surface et un vortex avec entraînement d'air sera plus rapide que sur le modèle.

\subsection{Entraînement d'air}

Il n'est pas possible de représenter à l'échelle la flottabilité d'une bulle d'air sur un modèle réalisé en appliquant la similitude de Froude. La bulle d'air prototype qui correspondrait à la bulle d'air observée sur le modèle (qui se crée spontanément sans que l'expérimentateur n'en maîtrise les dimensions) se déplacera plus vite jusqu'à la pompe dans la réalité que sur le modèle. Cette situation conduit à une approche conservatrice dans le traitement des observations faites au cours des expériences.

\subsection{Quelques remèdes}

Les coudes créent des gradients de vitesse [5] qui déséquilibrent le champ de vitesse. Placés à l'aspiration des pompes, ils induisent de la prérotation. On peut recommander d'éviter autant que possible cette pratique, il vaut mieux placer le coude (s'il est nécessaire) au refoulement. $\mathrm{Si}$, malgré tout, la prérotation persiste on peut redresser le champ de vitesse à l'aide d'aubes directrices installées dans le tube d'aspiration ou à son entrée.

Plus généralement, la démarche initiale du concepteur devrait être systématiquement de fonder son dessin sur les recommandations techniques usuelles, énoncées en particu- lier dans les fascicules de recommandations techniques de l'AFNOR n E 44202 et E 44203.

\section{VIII $\square$ OUVRAGES DE TRAITEMENT}

La cinétique chimie élémentaire nous explique qu'une réaction chimique ou biologique se déroule à une vitesse donnée. $\mathrm{Si}$ cette vitesse est inférieure à la vitesse à laquelle les espèces qui participent à la réaction se déplacent dans le fluide, la réaction est incomplète voire impossible. La physique explique aussi que la séparation densimétrique (sédimentation des corps plus denses que l'eau et flottation des corps moins denses que l'eau) ne peut s'opérer que si le fluide n'apporte pas d'énergie qui perturbe le phénomène : l'écoulement doit être lent et le champ de vitesse uniforme. En définitive, une bonne hydraulique est un préalable incontournable à l'obtention d'un ouvrage de traitement efficace.

Les équations empiriques ou semi-empiriques qui permettent de dessiner les ouvrages ne donnent qu'une description macroscopique de ces derniers. Par ailleurs, il n'existe pas de solutions analytiques aux équations qui régissent les écoulements dans les ouvrages de traitement.

Le modèle physique des réservoirs d'eau potable, des bassins de dessablement et, plus généralement, des ouvrages de traitement d'eau, permet de corriger le dessin initial jusqu'à obtenir un plan qui permet d'obtenir les caractéristiques de l'écoulement requises pour favoriser le déroulement des réactions chimiques mises en œuvre dans les traitements d'eau.

Les limites de l'expérimentation sur modèle sont atteintes dès lors que l'on met en place des sédiments dans le modèle pour évaluer l'efficacité du traitement. Les effets d'échelle interviennent alors dans les observations. On peut considérer que l'on quitte le domaine des modèles à fond fixe pour entrer dans le domaine des modèles de transport solide qui n'est pas dans le cadre du sujet de cet article.

\section{IX —OUVRAGES EN RÉSEAU}

Les ouvrages en réseau sont très variés : seuil déversoirs, puits de chute, nœud complexe raccordant plusieurs galeries. On leur accorde toujours une fonction critique : faciliter les écoulements et assurer ainsi la protection du public contre les inondations.

Le concepteur dessine ces ouvrages à l'aide d'équations qui ne décrivent pas les écoulements, en effet il n'existe pas de solutions analytiques aux équations de Navier Stokes (sauf dans quelques rares exceptions) et en particulier pas pour ces ouvrages spéciaux. Or, ceux-ci peuvent être le siège de phénomènes physiques qui contrecarrent notablement leur bon fonctionnement : mouvement de rotation en masse ou de vortex, entraînement d'air, turbulences dues aux mélanges des écoulements, etc...

Les mesures in situ montrent combien il est difficile de prédire le fonctionnement d'un déversoir et invalident bien souvent l'emploi d'une loi empirique connue pour prédire la capacité de délestage d'un seuil déversoir. Le modèle physique permet d'améliorer le dessin des zones d'approche du déversoir pour exploiter au maximum toute la largeur de lame déversante. En procédant à cette mise au point, l'expérimentateur optimise le dessin des ouvrages. En outre, le modèle permet de simuler des conditions de fonctionnement rares (forts débits, conditions aval particulières conduisant à un changement de régime de fonctionnement : dénoyé-noyé, etc...). En conséquence, l'étude sur modèle physique garantit le bon fonctionnement de l'ouvrage projeté, son dessin optimal et fournit la loi de déversement adaptée. 
L'entraînement d'air dans les puits de chute est un volet important de leur fonctionnement car, à certains débits, des phénomènes d'explosion peuvent se produire. La quantité d'air entraînée dans le puits dépend du régime d'écoulement dans le puits et du niveau d'eau dans le puits. Les structures d'entrée dans un puits jouent aussi un rôle particulier dans l'entraînement d'air, ces structures peuvent être :

- des déversoirs circulaires (barrages notamment);

- des entonnements à effet vortex ;

- des entrées en chute directe.

H.T. Falvey [6] consacre un chapitre de son ouvrage au problème d'entraînement d'air dans les puits de chute. On peut retenir de cet ouvrage qu'il est important de consacrer des études spécifiques à ce volet du fonctionnement d'un puits de chute. En effet, la capacité hydraulique d'évacuation du puits peut être nettement réduite par la constriction de la veine liquide due à la présence d'air. En conséquence, l'ouvrage peut ne pas disposer de la capacité théorique d'évacuation attendue. Il faut cependant être attentif au fonctionnement du modèle dans le cas des puits de chute car l'écoulement biphasique (air-eau) peut être soumis à des effets d'échelle qui biaisent ou invalident les observations.

\section{$\mathrm{X} \square$ CONCLUSION}

Le recours au modèle physique est une pratique de bon sens. Elle souligne le pragmatisme du maître d'ouvrage. Elle démontre la compétence du maître d'œuvre d'abord par sa capacité à juger (et à expliquer à son client) de la limite de la conception classique et de l'intérêt d'études détaillées ensuite par son aptitude à manipuler les outils employés dans les études sur modèles physiques.

\section{BIBLIOGRAPHIE}

[1] Dale Mosley J., Wayne Dillard C. (1995). - Designing a model pump station. Water environment \& technology. Avril 1995, pp. 46-50.

[2] CARLIER M. (1980). - Hydraulique générale et appliquée. Eyrolles. Collection de la Direction des Etudes et de la Recherche de EDF, Paris, 565 pages, annexes.

[3] Vergara Sanchez M.A. (1995). - Tecnicas de modelacion en hidraulica. Alfaomega grupo editor. Mexico. 294 pages.

[4] M.S. Yalin M.S. (1971). - Theory of Hydraulic Models. MacMillan Press, U.K, 266 pages.

[5] DS Miller D.S. (1990). - Internal flow systems. BHRA, Bedford, UK, second edition, 396 pages.

[6] HT. Falvey H.T. (1980). - Air-water flow in hydraulic structures. United States Department of the Interior. Water and Power Ressources Service (US Bureau of reclamation). Engineering monograph n 41,143 pages, annexes. 